\title{
Avaliação da amostragem com substituição parcial com duas medições sucessivas em floresta manejada no município de Paragominas, Pará.
}

\author{
Evaluation of sampling with partial replacement in two \\ successive occasions in a forest managed area in the \\ municipality Paragominas, Pará, Brazil.
}

\section{Luiz Otávio Mendonça Moniz Ribeiro ${ }^{1}$, José Natalino Macedo Silva ${ }^{1}$, Sylvio Péllico Netto ${ }^{2}$ e Waldenei Travassos de Queiroz ${ }^{1}$}

\begin{abstract}
Resumo
A legislação florestal brasileira estabelece que a intensidade de corte em florestas naturais seja planejada com base em estudos para determinar o incremento da floresta sob manejo. Nesse contexto, os processos de amostragem em ocasiões sucessivas assumem importância fundamental. Dentre esses, o processo de amostragem com repetição total (ART) utilizando unidades amostrais permanentes é o mais difundido e aplicado na Amazônia brasileira. Uma alternativa que tem sido pouco aplicada nos trópicos é a amostragem com substituição parcial (ASP). Este estudo buscou avaliar a aplicação da ASP como alternativa para monitorar o crescimento de florestas naturais na Amazônia brasileira. Os dados são oriundos de 30 unidades amostrais permanentes instaladas nas Unidades de Produção Anual 4 e 5 do Plano de Manejo Florestal Sustentável da Fazenda Rio Capim, município de Paragominas, Pará, cujo detentor é a empresa CKVB Florestal Ltda. As parcelas têm dimensões de 50 × 50 m (0,25 ha) e foram medidas nos anos de 2001 e 2004. Todos os indivíduos com o diâmetro a altura de $1,30 \mathrm{~m}$ do solo $(\mathrm{DAP}) \geq 10 \mathrm{~cm}$ foram registrados e utilizados no processamento. A ASP foi comparada com a ART utilizando os estimadores correspondentes, medidas de precisão e eficiências relativas, essas definidas como a razão entre as variâncias das médias. $O$ volume total na primeira ocasião utilizando a ASP foi de 219,15 $\mathrm{m}^{3} \mathrm{ha}^{-1} \pm 37,56 \mathrm{~m}^{3} \mathrm{ha}^{-1}$ e de $236,84 \mathrm{~m}^{3} \mathrm{ha}^{-1} \pm 39,69 \mathrm{~m}^{3} \mathrm{ha}{ }^{-1}$ na segunda ocasião. Para a ART, o volume total foi de $217,12 \mathrm{~m}^{3} \mathrm{ha}^{-1} \pm 43,96 \mathrm{~m}^{3} \mathrm{ha}^{-1}$ na primeira ocasião e de $232,32 \mathrm{~m}^{3} \mathrm{ha}^{-1} \pm 44,44$ $\mathrm{m}^{3}$ ha- ${ }^{-1}$ na segunda ocasião. O incremento periódico anual (IPA) foi de 4,66 $\mathrm{m}^{3} \mathrm{ha}^{-1} \mathrm{ano}^{-1} \mathrm{com}$ a ASP e de 4,68 $\mathrm{m}^{3} \mathrm{ha}^{-1} \mathrm{ano}^{-1} \mathrm{com}$ a ART. Utilizando a ASP estimou-se com maior eficiência o estoque volumétrico na primeira e na segunda ocasião. Por outro lado, o IPA da floresta foi estimado com maior eficiência com a ART. Não houve diferença estatística entre as estimativas das médias do volume para a primeira ocasião, segunda ocasião e incremento entre os processos. As estimativas geradas pelo processo de ASP mostraram-se competitivas quando comparadas às estimativas com o processo de ART, podendo, assim, ser usada como alternativa no monitoramento do crescimento florestal na Amazônia brasileira.
\end{abstract}

Palavras-chave: Amostragem em múltiplas ocasiões; Parcelas permanentes; Crescimento e produção florestal; Floresta tropical.

\begin{abstract}
1. Universidade Federal Rural da Amazônia - UFRA. Belém, PA, Brasil.

2. Universidade Federal do Paraná - UFPR. Curitiba, PR, Brasil.

* Autor correspondente: luizotavioflorestal@gmail.com
\end{abstract}

The Brazilian forest legislation establishes that the intensity of logging should be planned based on studies to determine volume increment of the forest under management. In this context, sampling processes on successive occasions are of utmost importance. Amongst these processes, continuous forest inventory (CFI) using permanent sampling plots is more widespread and applied in the Brazilian Amazon. An alternative less used in the tropics is the sampling with partial replacement (SPR). The present study aimed at evaluating the application of the SPR as an alternative process to monitoring growth and yield of natural forests in the Brazilian Amazon. Data from 30 permanent plots set space the Annual Production Units 4 and 5 of Rio Capim Forest Management Plan owned by the company CKVB Florestal Ltda. were used in the study. The forest management unit is located in Paragominas municipality in the state of Para, Brazil. The plot sizes are $50 \mathrm{mx} 50 \mathrm{~m}$ (0.25ha) and were measured in 2001 and in 2004 . All individuals with diameter at breast height (DBH) $\geq 10 \mathrm{~cm}$, were recorded and used in this study. SPR was compared to CFI using permanent sampling plots. Sampling precision and relative efficiency were used for comparisons. Relative efficiency was defined as the ratio of mean variances. The total volume estimated on the first occasion using SPR was $219,15 \mathrm{~m}^{3} \mathrm{ha} \mathrm{i}^{-1} \pm 37,56 \mathrm{~m}^{3} \mathrm{ha}^{-1}$ and $236,84 \mathrm{~m}^{3} \mathrm{ha}^{-1} \pm 39,69 \mathrm{~m}^{3} \mathrm{ha} \mathrm{a}^{-1}$ on the second occasion; for CFI the total volume was $217,12 \mathrm{~m}^{3} \mathrm{ha}^{-1} \pm 43,96 \mathrm{~m}^{3} \mathrm{ha}^{-1}$ 
for the first occasion and 232,32 $\mathrm{m}^{3} \mathrm{ha}^{-1} \pm 44,44 \mathrm{~m}^{3} \mathrm{ha} \mathrm{a}^{-1}$ for the second occasion. Periodic mean annual increment (PAI) was 4,66 m ha-1year ${ }^{-1}$ for SPR and 4,68 $\mathrm{m}^{3} \mathrm{ha}^{-1}$ year ${ }^{-1}$ for CFI. SPR was more efficient to estimate volume stock whereas CFI was more efficient to estimate the periodic annual increment (PAI). No statistical differences were found between estimates of the two processes. Estimates by SPR proved to be competitive when compared to $\mathrm{CFI}$ and therefore can be used as an alternative for monitoring growth and yield in the Brazilian Amazon.

Keywords: Continuous forest inventory; Permanent sample plots; Growth and yield; Tropical forest.

\section{INTRODUÇÃO}

O manejo florestal demanda grande quantidade de informação para a sua execução. A produção contínua e sustentável de produtos oriundos da floresta só é possível quando a exploração é vinculada à capacidade produtiva e às taxas de crescimento da floresta.

Nesse contexto, os inventários florestais assumem fundamental importância devido ao sucesso das decisões no manejo florestal estarem relacionadas com o grau de confiabilidade e da quantidade de informações obtidas sobre os recursos florestais (BICKFORD, 1963). Por meio dos inventários florestais sucessivos é possível avaliar o processo de evolução e de recomposição das características qualitativas e quantitativas da floresta, conhecendo-se, por exemplo, a dinâmica do crescimento de uma floresta pós-exploração madeireira ou definir os intervalos ideais de colheita dos produtos da floresta sob manejo sustentável (QUEIROZ, 2012).

Existem diferentes processos de inventários florestais sucessivos para monitorar a evolução da floresta. A escolha de um deles deverá basear-se nas características da floresta e nas condições encontradas na área. A legislação brasileira não especifica qual processo utilizar (BRASIL, 2006), cabendo ao manejador escolher a metodologia mais eficiente para obter informações de produção e crescimento da floresta.

Dentre os possíveis processos de combinação entre unidades amostrais permanentes e temporárias em inventários florestais sucessivos, a FAO (1981, p. 183) recomenda o uso da amostragem com substituição parcial (ASP): "Ao se planejar inventários florestais sucessivos os responsáveis por eles devem estruturá-los utilizando-se esse processo".

Aplicada pela primeira vez em inventários florestais contínuos por Bickford em 1956, o processo foi descrito de forma detalhada por Ware e Cunia (1962), considerando a relação que une duas medições sucessivas por meio das unidades amostrais permanentes como uma função linear simples. Nesse processo, parte das unidades amostrais medidas na primeira ocasião é remedida em uma segunda ocasião e a outra parte é substituída por novas unidades amostrais.

As vantagens da ASP foram comprovadas em alguns trabalhos (BICKFORD, 1956; BRENA, 1979; CUNIA, 1965; FRAYER; FURNIVAL, 1967; LIMA, 2010; RIBEIRO, 1978; SCOLFORO et al., 1981; WARE; CUNIA, 1962), tais como: menor custo em comparação à amostragem com repetição total das unidades amostrais (ART), essa tida como processo convencional, e versatilidade diante eventuais modificações nas condições entre as ocasiões de medição.

O conhecimento sobre o uso desse processo em florestas naturais na Amazônia é ainda incipiente, o que dificulta sua adoção. Assim, o presente trabalho teve como objetivo avaliar a aplicação da ASP em relação à ART a partir de suas eficiências estatísticas na obtenção dos volumes médios da primeira ocasião, segunda ocasião e crescimento, tendo em vista sua aplicação no manejo de florestas tropicais na Amazônia brasileira.

\section{MATERIAL E MÉTODOS}

\section{Área de estudo}

A pesquisa foi realizada nas Unidades de Produção Anual (UPA) 4, com 1.117,00 ha, e UPA 5, com 3.398,00 ha, do Plano de Manejo Florestal Sustentável (PMFS) da Fazenda Rio Capim, localizada no município de Paragominas, região nordeste do Estado do Pará (Figura 1). Utilizaram-se como critérios para selecionar as UPAs o número de unidades amostrais e o ano de medição e remedição. 

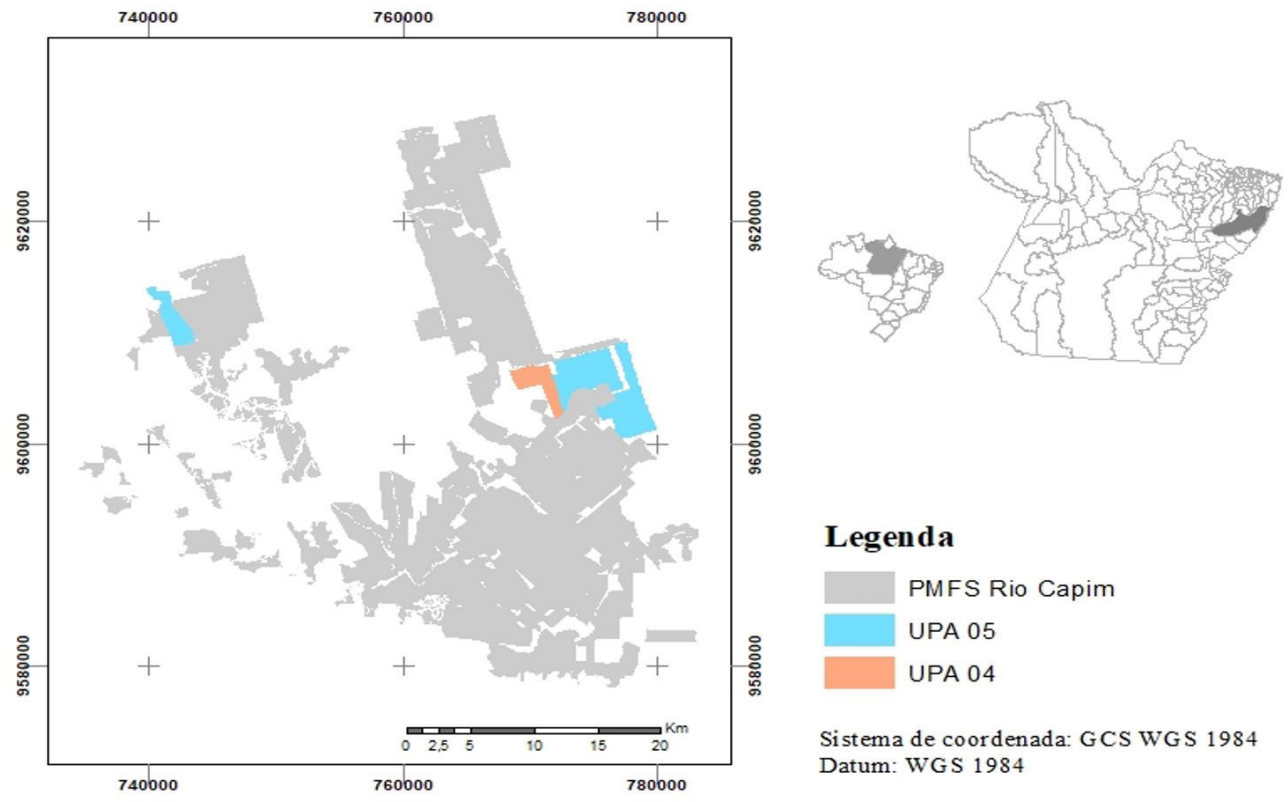

Legenda

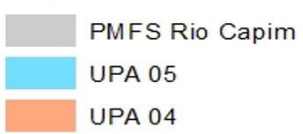

Sistema de coordenada: GCS WGS 1984 Datum: WGS 1984

FIGURA 1 - LOCALIZAÇÃO DAS UNIDADES DE PRODUÇÃO ANUAL 04 E 05, FAZENDA RIO CAPIM, PARAGOMINAS, PARÁ, BRASIL.

Figure 1 - Location of annual production units 04 e 05, Rio Capim Farm Paragominas, Pará, Brazil

A área de estudo é banhada pelas bacias dos rios Capim, que limita a área a Noroeste, e do Surubijú, que faz limite ao Sul. A topografia do terreno é plana à levemente ondulada, com altitude média em torno de 20 m. Localiza-se no Domínio Morfoestrutural dos Planaltos em Sequência Sedimentares Não Dobrados, com superfícies aplainadas na forma de extensos chapadões, limitados por planaltos dissecados sob forma de cristais e desenvolvida em rochas sedimentares (RODRIGUES et al., 2003). As principais classes de solos encontradas em Paragominas são: Argissolos Amarelos; Plintossolos; Gleissolos; Neossolos e Latossolos Amarelos de textura média e muito argilosa, que abrangem mais de $81,38 \%$ do município (RODRIGUES et al., 2003). Em geral, são solos com baixa fertilidade e alta saturação de alumínio (SCS, 2001).

O clima da região segundo a classificação Köppen é do tipo "Awi", isto é, tropical chuvoso com expressivo período de estiagem, caracterizado por temperatura média anual de $26^{\circ} \mathrm{C}$ e alta umidade relativa do ar (81\%). O período chuvoso estende-se de fevereiro até maio, com precipitação pluviométrica média anual de $1.700 \mathrm{~mm}$. O período de exploração madeireira coincide com o de menor disponibilidade hídrica, ocorrendo no período de julho a outubro.

A vegetação foi classificada de acordo com IBGE (2012), nos seguintes ambientes fitoecológicos: floresta ombrófila densa das terras baixas, floresta ombrófila densa aluvial e floresta ombrófila aberta das terras baixas. A tipologia nas UPAs 4 e 5 de acordo com a classificação do IBGE (2012) é floresta ombrófila densa das terras baixas, caracterizada por apresentar elevada biomassa, dossel fechado, árvores emergentes com altura variando de 30 a 50 metros, cujas as principais espécies comerciais encontradas são: Manilkara elata (Allemão ex Miq.) Monach (maçaranduba), Goupia glaba Aubl. (cupiúba), Caryocar villosum (Aubl.) Pers. (piquiá), Couratari sp. (tauari), Vouacapoua americana Aubl. (acapu), Tachigali glauca Tul. (tachi-preto), Lecythis lúrida (Miers) S.A.Mori (jarana), Alexa grandiflora Ducke (melancieira) e Parkia spp. (faveiras).

As UPAs 4 e 5 foram exploradas comercialmente no ano de 2000. O PMFS da Fazenda Rio Capim prevê um sistema silvicultural policíclico baseado na regeneração natural, com ciclos de corte de 30 anos. A intensidade média de exploração nas duas UPAs estudadas foi de $33 \mathrm{~m}^{3} \mathrm{ha}^{-1}$. Ressalta-se que a intensidade máxima de exploração não estava legalmente estabelecida a época da exploração.

\section{Coleta dos dados}

Os dados são provenientes do inventário florestal contínuo (IFC), a partir de 30 unidades amostrais distribuídas nas duas UPAs, sendo 9 pertencentes a UPA 4 e 21 a UPA 5, mensuradas nos anos de 2001 e 2004. 
A metodologia adotada para instalação e medição das unidades amostrais permanentes foi sugerida por Silva e Lopes (1984). Utilizaram-se, para o processamento, os dados referentes à variável quantitativa diâmetro a altura de 1,30 m do solo (DAP) de todos os indivíduos arbóreos com DAP superior ou igual a $10 \mathrm{~cm}$.

\section{Calculo do volume das unidades amostrais}

Os volumes individuais das árvores foram calculados com a seguinte equação desenvolvida por Baima et al. (2001) para o Campo Experimental da Embrapa situado no município de Moju, cuja fisionomia florestal assemelha-se à floresta estudada.

$$
\operatorname{Ln} V=-7,49337+2,086952 \ln D
$$

Em que:

$\mathrm{V}=$ volume com casca $\left(\mathrm{m}^{3}\right)$;

$\mathrm{D}=$ diâmetro do indivíduo a altura de 1,30 m do solo;

$\ln =$ logaritmo neperiano.

\subsection{Crescimento florestal}

O crescimento da floresta foi obtido pela diferença de produção entre a segunda ocasião (2004) e primeira ocasião (2001), definido como incremento periódico (IP). Para tal cálculo, excluiu-se a mortalidade e o ingresso, resultando no crescimento líquido da floresta no período estudado. Como não houve colheita entre os anos medidos, calculou-se o incremento periódico líquido, por meio da seguinte fórmula (CAMPOS; LEITE, 2013):

$$
I P_{l}=V_{2}-V_{1}+C-I n
$$

Em que:

$I P_{l}=$ Incremento periódico líquido;

$V_{1}=$ Volume total na primeira ocasião;

$V_{2}=$ Volume total na segunda ocasião;

$C$ = Colheita entre as ocasiões (neste caso $C=0$ );

In $=$ Volume de ingressos.

O incremento periódico anual (IPA), que representa a diferença de produção entre duas ocasiões dividida pelo período, em anos, foi calculado por meio da seguinte fórmula:

$$
\begin{gathered}
I P A_{l}=\frac{I P_{l}}{\text { periodo(anos })} \\
I P A_{l}=\text { Incremento periódico anual (líquido); }
\end{gathered}
$$

\section{Definição das subamostras e estimadores para os processos de amostragem em ocasiões sucessivas}

As unidades amostrais temporárias $(\mathrm{k})$ foram as sorteadas para fornecer os valores apenas da primeira ocasião (2001), desprezando os seus valores para a segunda (2004). As unidades permanentes (m) foram as sorteadas para fornecer os valores na primeira ocasião (2001) e na segunda (2004). As unidades amostrais novas (n) foram aquelas sorteadas para as estimativas da segunda ocasião (2004), ignorando seus valores de volume na primeira ocasião.

Os dados obtidos para cada subamostra foram submetidos ao teste de Shapiro-Wilk para verificar a suposição de normalidade dos dados. Para verificar a homogeneidade das variâncias dos volumes estimados na primeira e segunda ocasião, foi utilizado o teste F-máximo, ou teste de Hartley. As variâncias dos volumes estimados devem ser homogêneas para atender as pressuposições da análise de variância e permitir a comparação entre os métodos.

Os estimadores e notações utilizados foram retirados de Péllico Netto e Brena (1997) e Queiroz (2012) (Tabela 1). 
Ribeiro et al. - Avaliação da amostragem com substituição parcial com duas

medições sucessivas em floresta manejada no município de Paragominas, Pará.

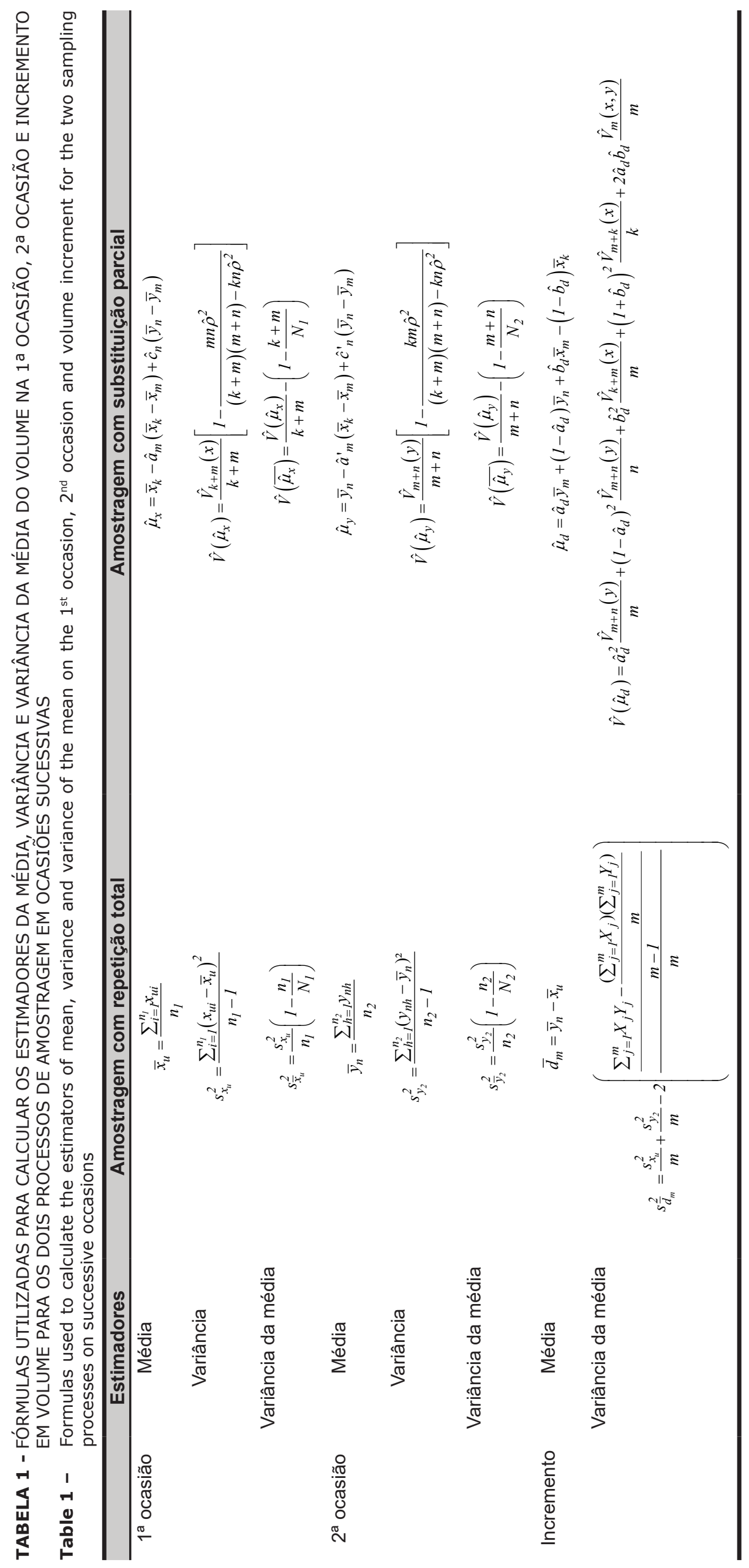


Em que,

$$
\begin{aligned}
& \hat{a}_{m}=\frac{m(m+n)}{(k+m)(m+n)-k n \hat{\rho}^{2}} \\
& \hat{c}_{n}=\frac{-m n \hat{B}_{x y}}{(k+m)(m+n)-k n \hat{\rho}^{2}}
\end{aligned}
$$

Tal que:

$$
\begin{gathered}
\hat{B}_{x y}=\frac{\hat{V}_{m}(x, y)}{\hat{V}_{m}(y)} \\
\hat{\rho}^{2}=\frac{\left[\hat{V}_{m}(x, y)\right]^{2}}{\hat{V}_{m}(x) \times \hat{V}_{m}(y)}
\end{gathered}
$$

Estimativa da variância não ASP da primeira ocasião para a subamostra k:

$$
\hat{V}_{k}(x)=\frac{\sum_{i=1}^{k} x_{i}^{2}-\frac{\left(\sum_{i=1}^{k} x_{i}\right)^{2}}{k}}{k-1}
$$

Estimativa da variância não ASP da primeira ocasião para a subamostra m:

$$
\hat{V}_{m}(x)=\frac{\sum_{i=k+1}^{k+m} x_{i}^{2}-\frac{\left(\sum_{i=k+1}^{k+m} x_{i}\right)^{2}}{m}}{m-1}
$$

Estimativa da variância não ASP da primeira ocasião para as subamostras $\mathrm{k}+\mathrm{m}$ :

$$
\hat{V}_{k+m}(x)=\frac{\sum_{i=l}^{k+m} x_{i}^{2}-\frac{\left(\sum_{i=l}^{k+m} x_{i}\right)^{2}}{k+m}}{k+m-1}
$$

Estimativa da variância não ASP da segunda ocasião para a subamostra m:

$$
\hat{V}_{m}(y)=\frac{\sum_{i=1}^{k+m} y_{i}^{2}-\frac{\left(\sum_{i=k+1}^{k+m} y_{i}\right)^{2}}{m}}{m-1}
$$

Estimativa da variância não ASP da segunda ocasião para a subamostra n:

$$
\hat{V}_{n}(y)=\frac{\sum_{i=k+m+1}^{k+m+n} y_{i}^{2}-\frac{\left(\sum_{i=k+m+1}^{k+m+n} y_{i}\right)^{2}}{n}}{n-1}
$$

Estimativa da variância não ASP da segunda ocasião paras as subamostras $n+m$ :

$$
\hat{V}_{m+n}(y)=\frac{\sum_{i=k+1}^{m+n} y_{i}^{2}-\frac{\left(\sum_{i=1}^{m+n} y_{i}\right)^{2}}{m+n}}{m+n-1}
$$

Estimativa da variância não ASP do incremento para a subamostra m:

$$
\hat{V}_{m}(x, y)=\frac{1}{m-1}\left[\sum_{i=k+1}^{k+m} x_{i} y_{i}-\frac{\left(\sum_{i=k+1}^{k+m} x_{i}\right)\left(\sum_{i=1}^{k+m} y_{i}\right)}{m}\right]
$$

E,

$$
\hat{c}_{n}^{\prime}=\frac{-m(k+m)}{(k+m)(m+n)-k n \hat{\rho}^{2}}
$$


Ribeiro et al. - Avaliação da amostragem com substituição parcial com duas

medições sucessivas em floresta manejada no município de Paragominas, Pará.

$$
\begin{aligned}
& \hat{a}_{m}^{\prime}=\frac{-k m \hat{B}_{y x}}{(k+m)(m+n)-k n \hat{\rho}^{2}} \\
& \hat{a}_{d}=\frac{m(k+m)+m n \hat{B}_{x y}}{(k+m)(m+n)-k n \hat{\rho}^{2}} \\
& \hat{b}_{d}=\frac{-m(m+n)+k m \hat{B}_{y x}}{(k+m)(m+n)-k n \hat{\rho}^{2}}
\end{aligned}
$$

Tal que:

$$
\hat{B}_{y x}=\frac{\hat{V}_{m}(x, y)}{\hat{V}_{m}(x)}
$$

Na Tabela 2 são apresentadas as notações utilizadas para exprimir as variáveis envolvidas nos cálculos para obtenção da estimativa do volume corrente na primeira ocasião, segunda ocasião, as estimativas das mudanças ocorridas na população florestal entre os dois períodos específicos e as estimativas das respectivas medidas de precisão, de acordo com Péllico Netto e Brena (1997) e Queiroz (2012).

TABELA 2 -NOTAÇÕES UTILIZADAS PARA EXPRIMIR AS VARIÁVEIS DOS PROCESSOS DE AMOSTRAGEM COM

\begin{tabular}{|c|c|}
\hline Variável & Descrição \\
\hline $\mathrm{m}$ & $\begin{array}{l}\text { Unidades de amostra permanentes, lançadas na } 1^{\text {a }} \text { ocasião e remedidas nas ocasiões subsequentes, } \\
\text { presente no processo de ART e ASP; }\end{array}$ \\
\hline $\mathrm{k}$ & $\begin{array}{l}\text { Unidades de amostra temporárias, lançadas independentemente na } 1^{a} \text { ocasião, adotada no processo } \\
\text { de ASP; }\end{array}$ \\
\hline u & $\begin{array}{l}\text { Unidades de amostra permanentes, lançadas independentemente na } 1^{\text {a }} \text { ocasião, adotada no processo } \\
\text { de ART; }\end{array}$ \\
\hline $\mathrm{N}$ & $\begin{array}{l}\text { Unidades de amostra temporárias novas e independentes, lançadas na } 2^{a} \text { ocasião, presente no } \\
\text { processo de ASP; No processo de ART, essas unidades amostrais são permanentes, ou seja, n = u. }\end{array}$ \\
\hline $\mathrm{N}_{1}$ & Número total de unidades amostrais na população estudada na 1ª ocasião; \\
\hline $\mathrm{n}_{1}$ & Número total de unidades amostrais medidas na $1^{\mathrm{a}}$ ocasião; \\
\hline $\mathrm{N}_{2}$ & Número total de unidades amostrais na população estudada na $2^{\mathrm{a}}$ ocasião; \\
\hline $\mathrm{n}_{2}$ & Número total de unidades amostrais medidas na $2^{\mathrm{a}}$ ocasião; \\
\hline $\mathrm{Y}_{\mathrm{nh}}$ & Volume na $2^{a}$ ocasião na unidade amostral $h$-ésima das novas, em que $(h=1,2,3, \ldots, n)$; \\
\hline $\mathrm{X}_{\mathrm{ui}}$ & Volume da $1^{\mathrm{a}}$ ocasião na i-ésima unidade amostral temporária ( $\left.\mathrm{i}=1,2,3, \ldots, \mathrm{k}\right)$ - ART; \\
\hline$\overline{\mathbf{x}}_{\mathbf{k}}$ & Estimativa sem tendência da média populacional para subamostrak $\left(\bar{X}_{k}\right)$ - ASP; \\
\hline$\overline{\mathbf{x}}_{\mathbf{m}}$ & Estimativa sem tendência da média populacional para subamostra m para a $1^{\mathrm{a}}$ ocasião $\left(\bar{X}_{m}\right)$ - ASP; \\
\hline$\overline{\mathbf{y}}_{\mathbf{m}}$ & Estimativa sem tendência da média populacional para subamostra m para a $2^{\mathrm{a}}$ ocasião $\left(\bar{Y}_{m}\right)-\mathrm{ASP}$; \\
\hline$\overline{\mathbf{x}}_{\mathbf{u}}$ & Estimativa sem tendência da média populacional para a $1^{\text {a }}$ ocasião $\left(\bar{X}_{u}\right)-\mathrm{ART}$; \\
\hline$\overline{\mathbf{y}}_{\mathbf{n}}$ & $\begin{array}{l}\text { Estimativa sem tendência da média populacional para a } 2^{\mathrm{a}} \text { ocasião }\left(\bar{Y}_{n}\right)-\mathrm{ART} \text {. Para ASP, representa a } \\
\text { estimativa sem tendência da média populacional para a subamostra } \mathrm{n}\left(\bar{Y}_{n}\right) ;\end{array}$ \\
\hline$\overline{\mathbf{d}}_{\mathrm{m}}$ & Estimativa sem tendência da média da diferença entre as duas ocasiões $\left(\bar{D}_{m}\right)$ - ART; \\
\hline $\mathbf{s}_{\mathbf{x}_{\mathrm{u}}}^{2}$ & Estimativa sem tendência da variância populacional para a $1^{\text {a }}$ ocasião $\left(S_{X_{u}}^{2}\right)$ - ART; \\
\hline $\mathbf{s}_{\mathbf{y}_{\mathbf{n}}}^{2}$ & Estimativa sem tendência da variância populacional para a $2^{\mathrm{a}}$ ocasião $\left(S_{Y_{n}}^{2}\right)$ - ART; \\
\hline$s_{\bar{x}_{u}}^{2}$ & Estimativa sem tendência da variância da média para a $1^{\mathrm{a}}$ ocasião $\left(S_{X_{u}}^{2}\right)$ - $\mathrm{ART}$; \\
\hline$s_{\bar{y}_{2}}^{2}$ & Estimativa sem tendência da variância da média para a $2^{\mathrm{a}}$ ocasião $\left(S_{Y_{n}}^{2}\right)$ - ART \\
\hline $\mathbf{s}_{\bar{d}_{m}}^{2}$ & Estimativa sem tendência da variância da média para o crescimento - ART; \\
\hline$\hat{\mathbf{a}}_{\mathbf{x y}}$ & Coeficiente de regressão linear de $\mathrm{X}$ sobre $\mathrm{Y}$; \\
\hline
\end{tabular}
SUBSTITUIÇÃO PARCIAL (ASP) E AMOSTRAGEM COM REPETIÇÃO TOTAL (ART)

Table 2 - Notation used to express the variables in the sampling processes with partial replacement and total replication 
TABELA 2 -CONTINUAÇÃO...

Table 2 - Continued...

\begin{tabular}{|c|c|}
\hline Variável & Descrição \\
\hline$\tilde{\mathbf{n}}$ & $\begin{array}{l}\text { Coeficiente de correlação da população entre os volumes das unidades amostrais permanentes } \\
\text { medidas nas duas ocasiões; }\end{array}$ \\
\hline$\hat{\mathbf{a}}_{\mathbf{y x}}$ & Coeficiente de regressão linear de Y sobre X; \\
\hline$\hat{V}_{k}(x)$ & Estimativa da variância não ASP da primeira ocasião para a subamostra k; \\
\hline$\hat{V}_{m}(x)$ & Estimativa da variância não ASP da primeira ocasião para a subamostra m; \\
\hline$\hat{V}_{k+m}(x)$ & Estimativa da variância não ASP da primeira ocasião para as subamostras $\mathrm{k}+\mathrm{m}$; \\
\hline$\hat{V}_{m}(y)$ & Estimativa da variância não ASP da segunda ocasião para a subamostra m; \\
\hline$\hat{V}_{n}(y)$ & Estimativa da variância não ASP da segunda ocasião para a subamostra n; \\
\hline$\hat{V}_{m+n}(y)$ & Estimativa da variância não ASP da segunda ocasião paras as subamostras $n+m$; \\
\hline$\hat{V}_{m}(x, y)$ & Estimativa da variância não ASP do incremento para a subamostra m; \\
\hline$\hat{\mu}_{x}$ & Estimativa sem tendência para o volume na $1^{\text {a }}$ ocasião - ASP; \\
\hline$\hat{V}\left(\hat{\mu}_{x}\right)$ & Estimativa sem tendência da variância populacional para a $1^{\text {a }}$ ocasião - ASP; \\
\hline$\hat{V}\left(\overline{\hat{\mu}_{x}}\right)$ & Estimativa sem tendência da variância da média para a $1^{\text {a }}$ ocasião - ASP; \\
\hline$\hat{\mu}_{y}$ & Estimativa sem tendência para o volume na $2^{\mathrm{a}}$ ocasião - ASP; \\
\hline$\hat{\boldsymbol{V}}\left(\hat{\boldsymbol{\mu}}_{\boldsymbol{y}}\right)$ & Estimativa sem tendência da variância populacional para a $2^{\mathrm{a}}$ ocasião - ASP; \\
\hline$\hat{V}\left(\overline{\hat{\mu}_{y}}\right)$ & Estimativa sem tendência da variância da média para a $2^{a}$ ocasião - ASP; \\
\hline$\hat{\mu}_{d}$ & Estimativa sem tendência da média da diferença entre as duas ocasiões - ASP; \\
\hline$\hat{V}\left(\hat{\mu}_{d}\right)$ & Estimativa sem tendência da variância da média para a diferença entre as duas ocasiões - ASP. \\
\hline
\end{tabular}

\section{Comparação entre os dois processos de amostragem em ocasiões sucessivas}

A comparação entre os processos de amostragem em ocasiões sucessivas foi realizada por meio da eficiência relativa entre os estimadores dos dois processos e pelo teste de comparação de médias, que indica se há diferença estatística entre as médias estimadas pelos dois processos.

A eficiência relativa de cada procedimento é calculada de acordo com Yamane (1974) citado por Brena (1979) e Scolforo et al. (1981) para trabalhos semelhantes.

$$
E R=\frac{\operatorname{VAR}\left(\hat{\theta}_{1}\right)}{\operatorname{VAR}\left(\hat{\theta}_{2}\right)}
$$

Em que:

ER = eficiência relativa;

$\operatorname{VAR}\left(\hat{\theta}_{1}\right)=$ variância da média do procedimento a ser comparado;

$\operatorname{VAR}\left(\hat{\theta}_{2}\right)=$ variância da média do procedimento tomado como base de comparação.

O teste t para amostras dependentes foi utilizado para comparar as médias estimadas pelos dois processos de amostragem em ocasiões sucessivas, a 95\% de probabilidade, verificando se há diferença estatística entre os valores obtidos.

\section{RESULTADOS E DISCUSSÃO}

Os resultados para a variável volume na primeira ocasião e na segunda ocasião das 30 unidades amostrais permanentes referentes às UPAs 4 e 5 estão apresentados na Tabela 3 . A média do volume por hectare (DAP $\geq 10 \mathrm{~cm}$ ) na primeira ocasião foi de $221,670 \mathrm{~m}^{3} \mathrm{ha}^{-1}$, com coeficiente de variação de $18,75 \%$ e desvio padrão de $\pm 41,561 \mathrm{~m}^{3} \mathrm{ha}^{-1}$. Para a segunda ocasião, a média foi de $236,860 \mathrm{~m}^{3} \mathrm{ha}^{-1}$, com coeficiente de variação de $17,74 \%$ e desvio padrão de $\pm 42,018 \mathrm{~m}^{3} \mathrm{ha}^{-1}$.

Na Tabela 4 são apresentados os volumes $\left(\mathrm{m}^{3} \mathrm{ha}^{-1}\right)$ das unidades amostrais sorteadas para compor o banco de dados da ASP. 
Ribeiro et al. - Avaliação da amostragem com substituição parcial com duas

medições sucessivas em floresta manejada no município de Paragominas, Pará.

TABELA 3 - VOLUMESDE MADEIRA COM CASCA $\left(\mathrm{m}^{3} \mathrm{ha}^{-1}\right)$ DAS UNIDADES AMOSTRAIS NA PRIMEIRA E SEGUNDA OCASIÃO, DAS UPAS 4 E 5, FAZENDA RIO CAPIM, PARAGOMINAS, PARÁ

Table 3- Plot volumes over bark $\left(\mathrm{m}^{3} \mathrm{ha}^{-1}\right)$ in first and second occasions, in UPA 4 and 5, at Rio Capim Forest management unit, Paragominas, Pará

\begin{tabular}{cccccc}
\hline No das Unidades & \multicolumn{2}{c}{ Volume $\left(\mathbf{m}^{3} / \mathbf{h a}\right)$} & $\mathbf{N}^{\circ}$ das Unidades & \multicolumn{2}{c}{ Volume $\left(\mathbf{m}^{3} / \mathbf{h a}\right)$} \\
\cline { 2 - 3 } amostrais & $\mathbf{2 0 0 1}$ & $\mathbf{2 0 0 4}$ & & $\mathbf{2 0 0 1}$ & $\mathbf{2 0 0 4}$ \\
\hline 1 & 252,069 & 263,439 & 16 & 155,242 & 171,996 \\
2 & 223,449 & 237,288 & 17 & 177,261 & 189,927 \\
3 & 200,167 & 215,938 & 18 & 232,027 & 245,587 \\
4 & 166,152 & 178,231 & 19 & 189,403 & 206,409 \\
5 & 144,655 & 162,718 & 20 & 248,317 & 268,652 \\
6 & 204,972 & 219,687 & 21 & 277,105 & 290,175 \\
7 & 189,420 & 203,135 & 22 & 270,722 & 288,601 \\
8 & 271,835 & 286,718 & 23 & 211,630 & 225,172 \\
9 & 253,138 & 265,147 & 24 & 212,605 & 228,591 \\
10 & 213,845 & 228,076 & 25 & 306,369 & 324,210 \\
11 & 260,185 & 280,855 & 26 & 239,556 & 255,589 \\
12 & 235,427 & 250,875 & 27 & 196,515 & 215,355 \\
13 & 204,526 & 222,667 & 28 & 238,997 & 252,665 \\
14 & 263,705 & 278,582 & 29 & 141,141 & 151,594 \\
15 & 203,947 & 218,592 & 30 & 265,712 & 279,353 \\
\hline
\end{tabular}

TABELA 4 - VOLUME DE MADEIRA COM CASCA $\left(\mathrm{m}^{3} \mathrm{ha}^{-1}\right)$ PARA AS UNIDADES AMOSTRAIS SORTEADAS PARA COMPOR O BANCO DE DADOS DO PROCESSO DE AMOSTRAGEM COM SUBSTITUIÇÃO PARCIAL (ASP) EM FLORESTA OMBRÓFILA DENSA MANEJADA, PARAGOMINAS, PARÁ

Table 4 - Timber volume over bark $\left(\mathrm{m}^{3} \mathrm{ha}^{-1}\right)$ for selected plots composing the database of the sampling with partial replacement process in a managed rainforest in Paragominas, Pará

\begin{tabular}{cccccc}
\hline U.a. & V 2001 & V 2004 & U.a. & V 2001 & V 2001 \\
\hline 11 & 260,185 & & 26 & 239,556 & 255,589 \\
24 & 212,605 & & 5 & 144,655 & 162,718 \\
1 & 252,069 & & 3 & 200,167 & 215,938 \\
10 & 213,845 & & 28 & 238,997 & 252,665 \\
19 & 189,403 & & 13 & 204,526 & 222,667 \\
17 & 177,261 & & 14 & 263,705 & 278,582 \\
6 & 204,972 & 219,687 & 21 & 277,105 & 290,175 \\
9 & 253,138 & 265,147 & 15 & 203,947 & 218,592 \\
29 & 141,141 & 151,594 & 25 & 306,369 & 324,210 \\
7 & 189,420 & 203,135 & 18 & & 245,587 \\
27 & 196,515 & 215,355 & 8 & & 286,718 \\
30 & 265,712 & 279,353 & 2 & & 237,288 \\
20 & 248,317 & 268,652 & 16 & & 171,996 \\
12 & 235,427 & 250,875 & 22 & & 288,601 \\
4 & 166,152 & 178,231 & 23 & & 225,172 \\
\hline
\end{tabular}

Na Tabela 5 mostram-se os volumes $\left(\mathrm{m}^{3} \mathrm{ha}^{-1}\right)$ das unidades amostrais sorteadas para compor o banco de dados da ART.

TABELA 5 -VOLUME DE MADEIRA COM CASCA $\left(\mathrm{m}^{3} \mathrm{ha}^{-1}\right)$ PARA AS UNIDADES AMOSTRAIS SORTEADAS PARA COMPOR O BANCO DE DADOS DO PROCESSO DE AMOSTRAGEM COM REPETIÇÃO TOTAL EM FLORESTA OMBRÓFILA DENSA MANEJADA, PARAGOMINAS, PARÁ

Table 5 - Timber volume over bark $\left(\mathrm{m}^{3} \mathrm{ha}^{-1}\right)$ for selected plots composing the database of continuous forest inventory process in a managed rain forest in Paragominas, Pará

\begin{tabular}{cccccc}
\hline U.a. & V 2001 & V 2004 & U.a. & V 2001 & V 2001 \\
\hline 14 & 272,618 & 278,394 & 8 & 209,343 & 213,812 \\
12 & 296,729 & 297,552 & 18 & 308,728 & 334,798 \\
15 & 272,637 & 219,760 & 11 & 283,968 & 295,403 \\
7 & 237,843 & 300,629 & 4 & 207,682 & 258,086 \\
1 & 330,553 & 340,916 & 2 & 253,116 & 253,488 \\
\hline
\end{tabular}


TABELA 5 - CONTINUAÇÃO...

Table 5 - Continued...

\begin{tabular}{cccccc}
\hline U.a. & V 2001 & V 2004 & U.a. & V 2001 & V 2001 \\
\hline 9 & 206,843 & 219,060 & 19 & 176,495 & 245,548 \\
20 & 237,892 & 268,348 & 13 & 324,932 & 293,233 \\
10 & 238,906 & 228,400 & 17 & 235,074 & 249,846 \\
6 & 175,233 & 176,792 & 3 & 236,009 & 217,534 \\
16 & 191,957 & 204,377 & 5 & 163,627 & 177,387 \\
29 & 141,141 & 151,594 & 25 & 306,369 & 324,210 \\
21 & 277,105 & 290,175 & 27 & 196,515 & 215,355 \\
\hline
\end{tabular}

\section{Estimativas para a variável volume $\left(\mathrm{m}^{3} \mathrm{ha}^{-1}\right)$ dos processos de inventário em ocasiões sucessivas}

Atendendo às condições citadas por Ware e Cunia (1962), cujo tamanho das amostras e as variâncias da população devem ser iguais nas duas ocasiões para possibilitar a comparação das variâncias das médias dos diferentes processos, procedeu-se o processamento dos dados para obter as estimativas da variável volume para os processos de inventário em ocasiões sucessivas em análise.

Na Tabela 6 estão apresentadas as principais estimativas do volume $\left(\mathrm{m}^{3} \mathrm{ha}^{-1}\right)$ para cada método de amostragem na primeira ocasião, segunda ocasião e o incremento no período de 2001 a 2004.

TABELA 6 - ESTATÍSTICA DOS PROCESSOS DE AMOSTRAGEM EM OCASIÕES SUCESSIVAS PARA VARIÁVEL VOLUME $\left(\mathrm{m}^{3} \mathrm{ha}^{-1}\right)$, APLICADOS EM UMA FLORESTA OMBRÓFILA DENSA, PARAGOMINAS, PARÁ. ART - AMOSTRAGEM COM REPETIÇÃO TOTAL; ASP - AMOSTRAGEM COM SUBSTITUIÇÃO PARCIAL

Table 6 - Statistics of sampling processes in successive occasions for variable volume $\left(\mathrm{m}^{3}\right.$ ha- 1$)$, applied to a rainforest in Paragominas, Pará. ART - Continuous Forest Inventory; ASP - Sampling with partial Replication

\begin{tabular}{|c|c|c|c|c|c|c|}
\hline \multirow{2}{*}{ Estimadores } & \multicolumn{2}{|c|}{ Primeira ocasião (2001) } & \multicolumn{2}{|c|}{ Segunda ocasião (2004) } & \multicolumn{2}{|c|}{ Incremento } \\
\hline & ART & ASP & ART & ASP & ART & ASP \\
\hline Média & $217,120^{\text {ns }}$ & $219,152^{\text {ns }}$ & $232,327^{\text {ns }}$ & $236,836^{\text {ns }}$ & $15,207^{\mathrm{ns}}$ & $17,683^{\text {ns }}$ \\
\hline Variância & 1932,548 & 1411,047 & 1974,714 & 1575,668 & & \\
\hline Variância da média & 80,523 & 58,794 & 82,279 & 65,653 & 0,318 & 3,422 \\
\hline Desvio padrão & 43,960 & 37,564 & 44,438 & 39,695 & & \\
\hline Erro padrão da média & 8,973 & 7,668 & 9,070 & 8,103 & 0,565 & 1,850 \\
\hline Coeficiente de variação (\%) & 20,25 & 17,37 & 19,13 & 16,761 & & \\
\hline Erro absoluto (+/-) & 18,563 & 15,862 & 18,764 & 16,762 & 1,168 & 3,700 \\
\hline Erro relativo (\%) & 8,55 & 7,24 & 8,08 & 7,08 & 7,68 & 20,92 \\
\hline
\end{tabular}

ns = não significativo ao nível de $95 \%$ de probabilidade.

O volume total na primeira ocasião utilizando a ASP foi de $219,152 \mathrm{~m}^{3} \mathrm{ha}^{-1} \pm 37,564 \mathrm{~m}^{3} \mathrm{ha}^{-1}$. Para a segunda ocasião, a estimativa do volume total por meio da ASP foi de $236,836 \mathrm{~m}^{3} \mathrm{ha}^{-1} \pm 39,695 \mathrm{~m}^{3} \mathrm{ha}^{-1}$.

Utilizando ART, o volume total para a primeira e segunda ocasião foi de $217,120 \mathrm{~m}^{3} \mathrm{ha}^{-1} \pm 43,960 \mathrm{~m}^{3} \mathrm{ha}^{-1} \mathrm{e}$ $232,327 \mathrm{~m}^{3} \mathrm{ha}^{-1} \pm 44,438 \mathrm{~m}^{3}$ ha ${ }^{-1}$, respectivamente.

O volume estimado pelos dois processos de inventário em ocasiões sucessivas para o ano de 2001 e 2004 (Tabela 6) condiz com o encontrado em florestas manejadas na Amazônia. No estado do Pará, principal produtor de madeira da Amazônia, os inventários diagnósticos utilizados como subsídios para elaboração de planos de manejo em áreas sob contratos de concessão florestal apresentaram volume total por hectare de variando de 186,82 a $296,74 \mathrm{~m}^{3} \mathrm{ha}^{-1}$, para árvores com DAP superior a $10 \mathrm{~cm}$ (BRASIL, 2017; PARÁ, 2015a;b, 2010). No Amazonas, Lima (2010) apresenta o resultado de diversos sítios florestais, com volume total por hectare variando de 172,92 a 388,53 $\mathrm{m}^{3} \mathrm{ha}^{-1}$ (DAP $\geq 10 \mathrm{~cm}$ ).

As médias, para a primeira e segunda ocasião, dos processos de amostragem em ocasiões sucessivas avaliados não diferem entre si $(\mathrm{p}>0,05)$. Entretanto, obteve-se maior eficiência relativa com a ASP na determinação do estoque volumétrico da primeira e segunda ocasiões, corroborando com os resultados relatados por Brena (1979) em povoamentos de Eucalyptus sp., Lima (2010) na Amazônia e Matos (2012) em Floresta Estacional Semidecidual. Essa maior eficiência pode ser explicada pelo fato da ASP utilizar valores das unidades amostrais das duas ocasiões para estimar o volume corrente, 
Ribeiro et al. - Avaliação da amostragem com substituição parcial com duas

medições sucessivas em floresta manejada no município de Paragominas, Pará.

ou seja, utiliza as subamostras temporárias, permanentes e novas para estimar o volume da primeira ocasião e da segunda ocasião.

A média do incremento líquido foi de $15,207 \mathrm{~m}^{3} \mathrm{ha}^{-1}$ para a ART e $17,683 \mathrm{~m}^{3} \mathrm{ha}^{-1}$ para a ASP no período de 2001 a 2004 (Tabela 6). Apesar de não haver diferença estatística na estimativa do incremento periódico ( $p>0,05)$, a ART teve eficiência relativa superior de 90\% comparado a ASP. A superioridade da ART também foi reportada por Brena (1979), Matos (2012) e Ribeiro (1978), na estimativa do crescimento florestal. Esse resultado é explicado em razão desse processo utilizar dados de todas as unidades amostrais remedidas para estimar o incremento, portanto, a ART é o processo mais recomendado para o monitoramento do crescimento florestal (SILVA et al., 2005; FELFILI et al., 2005).

No entanto, como a intensidade amostral foi semelhante para os dois processos, na ART foi utilizado maior número de unidades amostrais permanentes. Esse fato eleva o custo total do inventário, visto que o custo de instalação e remedição de unidades amostrais permanentes é superior ao custo de unidades amostrais temporárias (BRENA, 1979; LIMA, 2010; FRAYER; FURNIVAL, 1967; SCOLFORO, 1981). Dessa forma, não havendo diferença estatística na estimativa do incremento periódico, a ASP mostra-se adequada para ser adotada em inventários florestais contínuos na Amazônia.

Utilizando o limite inferior do intervalo de confiança para a média do incremento líquido, $14,039 \mathrm{~m}^{3} \mathrm{ha}^{-1}$ para a ART e 13,983 $\mathrm{m}^{3} \mathrm{ha}^{-1}$ para a ASP, o incremento periódico anual estimado foi de 4,679 $\mathrm{m}^{3} \mathrm{ha}^{-1} \mathrm{ano}^{-1}$ e 4,661 $\mathrm{m}^{3} \mathrm{ha}^{-1} \mathrm{ano}^{-1}$, respectivamente. Esses valores estão consentâneos aos padrões encontrados por outros autores, sendo superior aos encontrados por Silva et al. (1995), 1,8 $\mathrm{m}^{3} \mathrm{ha}^{-1} \mathrm{ano}^{-1}$, e Oliveira (2005), 1,9 $\mathrm{m}^{3} \mathrm{ha}^{-1} \mathrm{ano}^{-1}$, na Floresta Nacional do Tapajós-PA, para as espécies comerciais com DAP $\geq 20 \mathrm{~cm}$. Higuchi et al. (1997) encontraram incremento de 1,1 a 1,4 m $\mathrm{ha}^{-1}$ ano $^{-1}$ para espécies comerciais com DAP $\geq 10 \mathrm{~cm}$ no Amazonas. Os resultados foram semelhantes aos de 4,63 $\mathrm{m}^{3} \mathrm{ha}^{-1} \mathrm{ano}^{-1}$ observado por Souza et al. (2017) para todos os indivíduos com DAP $\geq 15 \mathrm{~cm}$, 18 anos após a exploração, e de Nascimento (2012), que encontrou, em três tratamentos distintos, incrementos de 4,15 $\mathrm{m}^{3} \mathrm{ha}^{-1} \mathrm{ano}^{-1}, 4,67 \mathrm{~m}^{3} \mathrm{ha}^{-1} \mathrm{ano}^{-1} \mathrm{e} 4,37 \mathrm{~m}^{3} \mathrm{ha}^{-1} \mathrm{ano}^{-1}$, para todos os indivíduos com $\mathrm{DAP} \geq 10 \mathrm{~cm}$. Ambos os estudos foram realizados em florestas manejadas na Amazônia Ocidental. Ao se comparar volumes de florestas em diferentes áreas da Bacia Amazônica, deve-se ter cautela devido à taxa de crescimento estar relacionada com as condições edáficas e climáticas dentro da referida bacia (QUESADA et al., 2012). Teixeira et al. (2007) relataram incremento de 5,60 $\mathrm{m}^{3} \mathrm{ha}^{-1} \mathrm{ano}^{-1}$ para todos os indivíduos com DAP $\geq 10 \mathrm{~cm}$, em florestas manejadas experimentalmente no Amazonas.

O número de unidades amostrais utilizadas pela empresa CKVB Florestal Ltda. foi suficiente para estimar o volume da primeira ocasião e segunda ocasião, para ambos os processos de amostragem, de acordo com a precisão requerida.

Apesar de ter gerado estimativa do incremento próxima do processo de ART, a ASP apresentou erro relativo superior a $10 \%$, sendo necessário aumentar o número de unidades amostrais para atingir o erro relativo almejado.

Mesmo com a versatilidade do processo de ASP, se adequando a modificações da precisão e estratificação e com a possibilidade de aumentar ou reduzir o número de unidades amostrais de cada subamostra, a escolha do processo a ser adotado deverá ser baseada nas necessidades e situações encontradas em campo (VANCLAY, 1992) e no orçamento disponível para realizar o monitoramento da floresta (SCOLFORO et al., 1981).

\section{CONCLUSÃO}

As estimativas geradas pelo processo de ASP mostraram-se competitivas quando comparadas às estimativas do processo de ART, podendo, se conveniente, substituir esse processo no monitoramento do crescimento florestal.

\section{REFERÊNCIAS BIBLIOGRÁFICAS}

BAIMA, A. M. V.; SILVA, S. M. A.; SILVA, J. N. M. A silvicultura tropical na Amazônia: Equações de volume para uma floresta tropical de terra-firme Moju-Pará. Belém: EMBRAPA Amazônia Oriental, 2001. p. $367-392$.

BICKFORD, C. A. On successive forest inventories. Proceedings of Society of American Foresters, p. 25-30, 1963. 
BICKFORD, C. A. Proposed design for continuous forest inventory: a system of perpetual Forest Survey for the Northeast. Ed: Cumberland Falls, Ky. U.S. Service Eastern Techniques Meeting Forest Survey, 1956. p. 8-13.

BRASIL. Serviço Florestal Brasileiro. Plano de Manejo Sustentável da Unidade de Manejo Florestal II - Concessão Florestal na Floresta Nacional de Altamira. Belém. 2017. 120 p. Não publicado.

BRASIL. Ministério do Meio Ambiente. Instrução Normativa No 5, de 11 de dezembro de 2006. Diário Oficial da República Federativa do Brasil, Brasília, DF, p. 155, Seção 1, 13 de dezembro de 2006.

BRENA, D. A. Comparação dos métodos de inventários florestais sucessivos em relação à amostragem com repetição parcial, aplicados em uma população estratificada. 1979, 127 p. Dissertação (Mestre em Ciências) - Universidade Federal do Paraná, Curitiba, 1979.

CAMPOS, J. C. C.; LEITE, H. G. Mensuração florestal: perguntas e respostas. 2 ed. Viçosa: Editora UFV, 2006. $470 \mathrm{p}$.

CUNIA, T. Continuous forest inventory, partial replacement of samples and multiple regression. Forest Science, v, 11, n 4, p.480-502, 1965.

FAO - FOOD AND AGRICULTURE ORGANIZATION. Manual of Forest inventory: with special reference to mixed tropical forest. Roma: FAO, 1981. 200 p.

FELFILI, J. M.; CARVALHO, F. A.; HAIDAR, R. F. Manual para o monitoramento de parcelas permanentes nos biomas cerrado e pantanal. Brasília: Universidade de Brasília, Departamento de Engenharia Florestal, 2005. 60 p.

FRAYER, W. E.; FURNIVAL, G. M. Are a change estimates from sampling with partial replacement. Forest Science, v. 13, n. 1, p 72-77, 1967.

HIGUCHI, N.; SANTOS, J.; RIBEIRO, R. J.; FREITAS, J. V.; VIEIRA, G.; COIC, A. R.; MINETTE, L. J. Crescimento e incremento de uma floresta Amazônica de terra-firme manejada experimentalmente. In: INPA. Biomassa de Nutrientes Florestais. Manaus: INPA/DFID, 1997. p 89-132.

IBGE - INSTITUTO BRASILEIRO DE GEOGRAFIA E ESTATÍSTICA. Manual técnico da vegetação brasileira: sistema fitogeográfico, inventário das formações florestais e campestres, técnicas e manejo de coleções botânicas, procedimentos para mapeamentos. 2.ed.Rio de Janeiro: IBGE- Diretoria de Geociências, 2012. 271 p. (Manuais técnicos em geociências 1).

LIMA, A. J. N. Avaliação de um sistema de inventário florestal contínuo em áreas manejadas e não manejadas do Estado do Amazonas (AM). 2010. 183 p. Tese (Ciências de Florestas Tropicais). Instituto Nacional de Pesquisa da Amazônia, Manaus, AM, 2010.

MATOS, L. M. S. Avaliação de métodos de amostragem em ocasiões sucessivas aplicados no manejo de florestas inequiâneas. 2012. 72 p. Dissertação (Mestrado em Ciência Florestal). Universidade Federal de Viçosa, Viçosa, 2012.

OLIVEIRA, L. C. Efeito da exploração da madeira e de diferentes intensidades de desbaste sobre a dinâmica da vegetação de uma área de 136 ha na Floresta Nacional do Tapajós. 2005. 183 p. Tese (Doutorado Recursos Florestais) - Universidade de São Paulo, Piracicaba, 2005.

PARÁ. Secretaria de Meio Ambiente e Sustentabilidade. Plano de Manejo Florestal Sustentável de Uso Múltiplo - Concessão Florestal da Floresta Nacional Crepori. Belém: Brasadoc Timber Comércio de Madeiras Ltda, 2015a. 206 p. Não publicado.

PARÁ. Secretaria de Meio Ambiente e Sustentabilidade. Resumo executivo do inventário florestal amostral. Belém, 2015b. 12 p. Não publicado.

PARÁ. Secretaria de Meio Ambiente e Sustentabilidade. Inventário florestal diagnóstico do conjunto de glebas estaduais Mamuru-arapiuns - Pará. Santarém, 2010. 135 p. (Relatório Final Não publicado).

PÉLLICO NETTO, S.; BRENA, D. A. Inventário florestal. Curitiba: Ed. UFPR, 1997. 316 p.

QUEIROZ, W. T. Amostragem em inventário florestal. Ed. UFRA. Belém: Universidade Federal Rural da Amazônia, 2012. 441 p. 
Ribeiro et al. - Avaliação da amostragem com substituição parcial com duas

medições sucessivas em floresta manejada no município de Paragominas, Pará.

QUESADA, C. A.; PHILLIPS, O. L.; SCHWARZ, M.; CZIMCZIK, C. I.; BAKER, T. R.; PATIÑO, S.; FYLLAS, N. M.; HODNETT, M.G.; HERRERA, R.; ALMEIDA, S.; ALVAREZ, E. D.; ARNETH, A.; ARROYO, L.; CHAO, K. J.; DEZZEO, N.; ERWIN, T.; FIORE, A. D.; HIGUCHI, N.; CORONADO, E. H.; JIMENEZ, E. M.; KILLEEN, T.; LEZAMA, A. T.; LLOYD, G.; LÓPEZ-GONZÁLEZ, G.; LUIZÃO, F. J.; MALHI, Y.; MONTEAGUDO, A.; NEILL, D. A.; VARGAS, P. N.; PAIVA, R.; PEACOCK, J.; PEÑUELA, M. C.; CRUZ, A. P.; PITMAN, N.; PRIANTE, N.; PRIETO, A.; RAMÍREZ, H.; RUDAS, A.; SALOMÃO R.; SANTOS, A. J. B.; SCHMERLER, J.; SILVA, N.; SILVEIRA, M.; VÁSQUEZ, R.; VIEIRA, I.; TERBORGH, J.; LLOYD, J. Basin-wide variations in Amazon forest structure and function are mediated by both soils and climate. Biogeosciences, v. 9, n. 6, p. 2203 - 2246, 2012.

RIBEIRO, J. C. Análise da amostragem com repetição parcial em relação a outros procedimentos de inventários florestais sucessivos. 1978. 99 p. Dissertação (Mestre em Ciência Florestal) - Universidade Federal do Paraná, Curitiba, 1978.

RODRIGUES, T. E.; SILVA, R. C.; SILVA, J. M. L.; OLIVEIRA JÚNIOR, R. C.; GAMA, J. R. N.; VALENTE, M. A. Caracterização e classificação dos solos no município de Paragominas, Estado do Pará. Belém: Empresa Brasileira de Pesquisa Agropecuária, Documentos, 162. 51 p. 2003.

SCOLFORO, J. R. S.; PAULA, F. N.; ASPIAZÚ, C.; CONDÉ, A. R. Análise comparativa dos procedimentos de inventários florestais repetidos em povoamentos de Eucalyptus sp. na região de Bom Despacho. Revista Árvores, Viçosa, v. 6, n. 1, p. 56-72, 1981.

SILVA, J. N. M.; LOPES, J. C. A.Inventário florestal contínuo em florestas tropicais: a metodologia pela EMBRAPA - CPATU na Amazônia brasileira. Belém: Empresa Brasileira de Pesquisa Agropecuária, Documentos, 33.36 p. 1984.

SILVA, J. N. M.; CARVAlHO, J. O. P.; LOPES, J. C. A.; ALMEIDA, B. F.; COSTA, D. H. M. Growth and yield of a tropical rain forest in the Brazilian Amazon 13 years after logging. Forest Ecology and Management, n. 71, p. 267-274, 1995.

SILVA, J. N. M.; LOPES, J. C. A.; OliVEIRA, L. C.; SILVA, S. M. A.; CARVALHO, J. O. P.; COSTA, D. H. M.; TAVARES, M. J. M. Diretrizes para instalação e medição de parcelas permanentes em florestas naturais da Amazônia Brasileira. Belém: Embrapa Amazônia Oriental, 2005. p. 68.

SOUZA, M. A. S.; AZEVEDO, C. P.; SOUZA, C. R.; FRANÇA, M.; VASCONCELOS NETO, E. L. Dinâmica e produção de uma floresta sob regime de manejo sustentável na Amazônia central. Floresta, Curitiba, v. 47, n. 1, p. 55-63, 2017.

TEIXEIRA, L. M.; CHAMBERS, J. Q.; SILVA, A. R.; LIMA, A. J. N.; CARNEIRO, V. M. C.; SANTOS, J.; HIGUCHI, N. Projeção da dinâmica da floresta natural de Terra-firme, região de Manaus-AM, com uso da cadeia de transição probabilística de Markov. Acta Amazônica, Manaus, v. 37, n. 3, p. 377-384, 2007.

VANCLAY, J. K. Permanent plots for multiple objectives: Defining goals and resolving conflicts. In: LUND, H. G.; PÄIVINEN, R.; THAMMINCHA, S. (ed). Remote and permanent plot techiniques for world forest monitoring. Pattaya: Wacharakitti International Workshop, 1992. p. 157-163.

WARE, K. D.; CUNIA, T. Continuous forest inventory with partial replacement of samples. Ed: Washington, Society of American Foresters, Forest Science, Monograph, 40 p, 1962.

YAMANE, T. Estadistica. Harla: Ed. México, 1974. 573 p.

Recebido em: 30/07/2018

Aceito em: 21/03/2019 